\title{
Induction of Tolerance to Human Arylsulfatase A in a Mouse Model of Metachromatic Leukodystrophy
}

\author{
Ulrich Matzner, ${ }^{1}$ Frank Matthes, ${ }^{1}$ Eva Herbst, ${ }^{2}$ Renate Lüllmann-Rauch, ${ }^{2}$ Zsuzsanna Callaerts-Vegh, ${ }^{3}$ \\ Rudi D'Hooge, ${ }^{3}$ Cecilia Weigelt, ${ }^{4}$ Carl Eistrup, ${ }^{4}$ Jens Fogh, ${ }^{4}$ and Volkmar Gieselmann ${ }^{1}$ \\ ${ }^{1}$ Institut für Physiologische Chemie, Rheinische Friedrich-Wilhelms-Universität, Nussallee 11, D-5315 Bonn, Germany; ${ }^{2}$ Anatomisches \\ Institut, Christian-Albrechts-Universität, Olshausenstr. 40, D-24098 Kiel, Germany; ${ }^{3}$ Laboratory of Biological Psychology, Department \\ of Psychology, University of Leuven, Tiensestraat 102, B-3000 Leuven, Belgium; ${ }^{4}$ Zymenex A/S, Roskildevej 12C, DK-3400 Hillerød,
} Denmark and Dalenum 13, S-18170 Lidingö, Sweden

\begin{abstract}
A deficiency of arylsulfatase A (ASA) causes metachromatic leukodystrophy (MLD), a lysosomal storage disorder characterized by accumulation of sulfatide, a severe neurological phenotype and early death. The efficacy of enzyme replacement therapy (ERT) has previously been determined in ASA knockout (ASA-/-) mice representing the only available animal model for MLD. Repeated intravenous injection of human ASA (hASA) improved the nervous system pathology and function, but also elicited a progressive humoral immune response leading to treatment resistance, anaphylactic reactions, and high mortality. In contrast to ASA-/- mice, most MLD patients express mutant hASA which may entail immunological tolerance to substituted wildtype hASA and thus protect from immunological complications. To test this notion, a cysteine-to-serine substitution was introduced into the active site of the hASA and the resulting inactive hASA-C69S variant was constitutively expressed in ASA-/- mice. Mice with subto supranormal levels of mutant hASA expression were analyzed. All mice, including those showing transgene expression below the limit of detection, were immunologically unresponsive to injected hASA. More than 100-fold overexpression did not induce an overt new phenotype except occasional intralysosomal deposition of minor amounts of glycogen in hepatocytes. Furthermore, long-term, low-dose ERT reduced sulfatide storage in peripheral tissues and the central nervous system indicating that high levels of extracellular mutant hASA do not prevent cellular uptake and lysosomal targeting of substituted wildtype hASA. Due to the tolerance to hASA and maintenance of the MLD-like phenotype, the novel transgenic strain may be particularly advantageous to assess the benefit and risk of long-term ERT.
\end{abstract}

Online address: http://www.molmed.org

doi: 10.2119/2007-00063.Matzner

\section{INTRODUCTION}

Metachromatic leukodystrophy (MLD) is a lysosomal storage disease (LSD) which is caused by a functional deficiency of the lysosomal enzyme arylsulfatase A (ASA; E.C. 3.1.6.8.) (1). ASA catalyzes the desulfation of the acidic sphingolipid 3-O-sulfogalactosylceramide (sulfatide) which is transported to the lysosomal compartment for degradation. Lack of ASA results in a block of sulfatide hydrolysis and the deposition of sulfatide in intralysosomal storage granules. Sulfatide storage in oligodendrocytes and Schwann cells leads to demyelination of the peripheral and central nervous system, a variety of rapidly de- teriorating neurological symptoms and premature death.

The phenotype of ASA-/- mice recapitulates the early phases of MLD, but does not proceed to the advanced stages, which are characterized by widespread demyelination, severe ataxia, blindness, quadriplegia, and loss of volitional movements $(1,2)$. Despite the relatively mild phenotype, the knockout mouse turned out to be a valuable model to test experimental therapies (3).

Among European MLD patients, only three ASA mutations occur with high frequency and in only one of them (allele I) ASA is immunologically undetectable (4). Around 6 percent of the MLD pa-

Address correspondence and reprint requests to Ulrich Matzner, Institut für Physiologische Chemie, Rheinische Friedrich-Wilhelms-Universität, Nussallee 11, D-5315 Bonn, Germany. Phone: 49-228-734744; Fax: 49-228-732416; Email: matzner@institut.physiochem.uni-bonn.de. Submitted June 13, 2007; Accepted for publication July 9, 2007.

tients are homozygous for this allele. Thus, around 94 percent of all MLD patients are not deficient for ASA, but express an ASA polypeptide, albeit on a low level or on a normal level, but with reduced specific activity or stability. ASA-/- mice, however, do not express any residual enzyme (2). Thus, besides the mild phenotype the complete lack of ASA expression is a major difference between the murine and most forms of the human disease.

The presence of residual enzyme may have important consequences for therapies which are based on the substitution of wildtype ASA. Under such regimens the mouse is confronted with a novel polypeptide, which, because it is unknown to the immune system, acts as an antigen and elicits an immune response. Human patients with cross-reacting immunological material, on the contrary, are likely to be protected from severe 
immune complications if the hASA mutation(s) does not cause loss or gross abnormalities in the folding of epitopes inducing self-tolerance during the late fetal and early postnatal period (5).

The ASA-/- mouse model has been used previously to determine therapeutic efficacy and immune-mediated side effects of ERT. High-dose, short-term treatment using four injections of $20 \mathrm{mg}$ hASA per kg body weight reduced sulfatide storage in kidney and peripheral nerves by approximately two thirds and in brain by roughly one third (6). In addition, treatment normalized the peripheral nerve conduction and improved the motor coordination and rotarod performance. Immunological complications elicited by repeatedly substituted hASA included development of resistance to treatment and progressive hypersensitivity reactions (Matzner et al., submitted). In fact, treatment for more than four weeks was not possible because the majority of animals died from anaphylactic reactions (6).

Immunological problems during ERT are not unique to the ASA-/- model, but have been observed in knockout mouse models for other LSDs as well $(7,8)$. Due to profound immune responses to therapeutic enzyme, knockout mice are of limited value to assess the full clinical benefit and risk of enzyme substitution therapies. Several approaches to circumvent this limitation have been investigated. Constitutive expression of inactive enzyme from a stably integrated transgene was effective in inducing tolerance to therapeutic enzyme in MPS IVa and VII mice (9-11). Here we describe a similar approach using transgenic expression of an active site mutant of the hASA polypeptide.

\section{MATERIALS AND METHODS}

\section{Materials}

Chemicals were obtained from SigmaAldrich (Deisenhofen, Germany), Merck (Darmstadt, Germany) or CalbiochemNovabiochem (Schwalbach, Germany). Secondary antibodies were from Jackson IR Laboratories (West Grove, PA, USA).
Recombinant hASA was purified as described (6). The eukaryotic expression plasmid pTVC was kindly provided by William S. Sly (St. Louis, MO, USA). Enzymes used for DNA modification were from Fermentas (St. Leon Rot, Germany). Oligonucleotides were from MWG Biotech (Ebersberg, Germany).

\section{Enzyme Analyses}

Activities of lysosomal enzymes were measured as described $(12,13)$. Concentrations of wildtype hASA and hASAC69S were determined by an indirect sandwich ELISA (14). Protein concentrations were measured with the Dc assay from BioRad (Hercules, CA, USA).

\section{In vitro Mutagenesis}

Bluescript-HT14/CP8 containing the full length wildtype hASA cDNA (15) was used as a template for PCR-based in vitro mutagenesis utilizing the QuikChange Site-Directed Mutagenesis Kit (Stratagene) and the mutagenic primers ASA-C69S-s (5'-GTGCCTGTGTCTCTGAGTACTCCCTCTAGGGCCGCC-3') and ASA-C69S-as (5'-GGCGGCCCTAGAGGGAGTACTCAGAGACA CAGGCAC-3'). PCR-amplification changed the coding information for cysteine-69 to serine, destroyed an ApaLI restriction site and introduced a ScaI site (Figure 1A). The mutant hASA-C69S coding sequence was ligated in both orientations into the pTVC backbone as a BamHI fragment (Figure 1B).

\section{Cell Culture Experiments}

Baby hamster kidney (BHK) cells were maintained, transfected, and immunostained as described $(16,17)$.

\section{Transgenic Mice}

Transgenic mice were generated at the Karolinska Centre for Transgene Technology (KCTT) by the pronuclear injection technique. For that purpose the XhoIexcised expression cassette of pTVC hASA-C69S was injected into fertilized oocytes from Bl6 wildtype mice. Among 34 live borns, two expressed hASA-C69S in serum. Transgene integration was verified by PCR of genomic DNA isolated from tail biopsies using the primer pair ASA-27 (5'-ATGACCTCATGCCGACGCCCAGCGCCAGG-3') and ASA-8 (5'-GTCAGAGAGCTGGAGGCGTG-3') and appropriate PCR conditions (32 cycles of $94^{\circ} \mathrm{C}$ for $30 \mathrm{~s}, 56^{\circ} \mathrm{C}$ for $30 \mathrm{~s}$, and $72^{\circ} \mathrm{C}$ for one minute, $0.5 \mathrm{U}$ Taq polymerase, $0.2 \mathrm{mM}$ dNTPs, $2 \mathrm{mM} \mathrm{MgCl}{ }_{2}$, ten percent dimethylsulfoxide, $10 \mathrm{pmol}$ of each primer, total volume of $25 \mu \mathrm{L}$ ). A male founder was mated with ASA-/females (2) and transgene-positive F1 offspring were internally crossed. Transgene-positive F2 pups were genotyped for the murine ASA (mASA) knockout allele using ASA-anti (5'- TGACCCAGGCCTTGTTCCCAT-3'), ASA-sense (5'TAGGGTGGAAGTTACCCTAGA-3') and ASA-neo (5'- GGAGAGGCTATTCGGCTATGA C-3') as primers and the PCR conditions described earlier. Appropriate F2 mice were mated to produce F3 mice of the genotype combinations hASAC69S + (transgenic ASA-/- mice being hemizygous or homozygous for the transgene), mASA-/- hASA-C69S-/- (nontransgenic ASA-/- mice), mASA+/+ hASA-C69S-/- (wildtype mice) and mASA+/+ hASA-C69S+. Mice of the F2 and F3 generation were used throughout the study.

Mice were maintained on a $12: 12 \mathrm{~h}$ light/dark cycle according to German animal protection laws. All experimental procedures were approved by the regional authorities (Bezirksregierung Köln, reference number 50.203.2-BN 24, 1/05).

\section{Treatment and Phenotypic Assessment}

Recombinant hASA was intravenously injected into the tail vein of seven- to nineweek-old mice. For the determination of hASA-C69S concentrations, for lipid analysis and for histological examinations, mice, blood, and tissue samples were processed as described $(6,17)$. Swimming velocity was recorded as reported (18). The concentration of $\alpha$-hASA antibodies in mouse serum was determined by immunoprecipitation (19). Statistical calculations were performed using InStat version 3.06 (GraphPad, San Diego, CA, USA). 


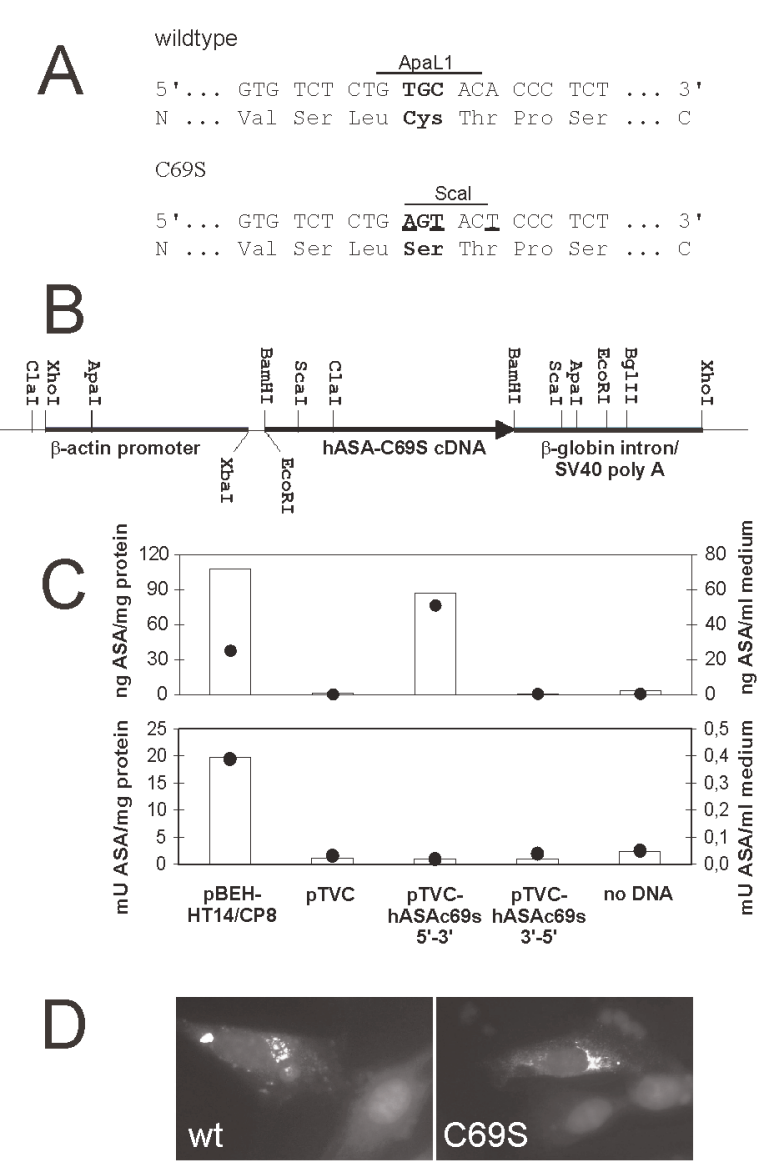

Figure 1. In vitro mutagenesis of the wildtype hASA CDNA and expression of hASA-C69S in cultured cells. (A) Exchange of three nucleotides (underlined) of the wildtype hASA cDNA resulted in the substitution of cysteine by serine at position 69 (bold letters), the loss of an ApaLI restriction site and the gain of a Scal site. (B) The hASA-C69S CDNA was cloned under the control of the chicken $\beta$-actin promoter of the eukaryotic expression vector pTVC. (C) BHK cells were transiently transfected with pTVC harboring the hASA-C69S in 5'$3^{\prime}$ or, as a control, in 3'-5' orientation. For additional controls, PBEH-HT14/CP8 bearing the wildtype hASA cDNA under the control of the SV40 promoter (15), the empty TVC plasmid (pTVC) or no DNA was used. ASA was quantified by ELISA (upper panel) or activity assays (lower panel) using cell homogenates (bars) and conditioned medium (closed circles). (D) Immunofluorescence of wildtype (wt) and mutant hASA (C69S) in BHK cells transiently transfected with PBEH-HT14/CP8 and PTVC hASA-C69S 5'-3', respectively. The red perinuclear hASA staining of the original color images is visible as a bright signal after conversion into the black and white image. The DAPI staining of the nuclei appears gray.

\section{RESULTS}

\section{In vitro Mutagenesis of the hASA cDNA}

The active site of eukaryotic sulfatases harbors a conserved cysteine residue which is essential for catalytic activity (20). To destroy the activity of wildtype hASA, the coding information for the active site cysteine was changed to serine (Figure 1A). The mutated cDNA was cloned into the eukaryotic expression vector pTVC (Figure 1B) and the construct, pTVC hASA-C69S, was used for transient transfection of BHK cells. High overexpression of hASAC69S polypeptide, but no increase of the sulfatase activity, could be detected in cell homogenates and medium (Figure 1C). Immunofluorescence experiments revealed normal lysosomal sorting of intracellularly retained hASA-C69S (Figure 1D).

\section{Transgenic Expression of Inactive hASA in ASA-/- Mice}

Mice which were deficient for the murine ASA and transgenic for hASAC69S were generated. The hASA-C69S serum concentration of these mice varied between levels below detection $(<0.01 \mu \mathrm{g} /$ $\mathrm{mL}$ ) and $3 \mu \mathrm{g} / \mathrm{mL}$ (Figure 2A). Tissue levels were highest in brain, intermediate in spinal cord, and lowest in peripheral nerves (plexus brachialis, sciatic nerve), kidney, and liver (Figure 2B). Immunostaining revealed high hASA-C69S levels in neuronal cells throughout the CNS (Figure 3A) and only low levels in liver (Figure 3B). Some hASA-C69S-positive liver cells could be identified as Kupffer cells by co-immunostaining with antibodies to F4/80, a macrophage-restricted surface marker. No expression was found in hepatocytes.

\section{Sulfatide Storage and Neuromotor Coordination in Transgenic ASA-/- Mice}

TLC and histological analyses of six-month-old transgenic ASA-/mice revealed sulfatide storage patterns which were indiscernible from those of conventional, age-matched ASA-/- mice (Figure 2C, Figure 3C,D). Storage was most prominent in neuronal cells and phagocytes of the spinal cord and in certain nephron segments of the kidney (Figure 3 C,D) $(21,22)$. To determine motor coordination impairments the swimming velocity was analyzed. Compared with wildtype controls, the mean velocity of transgenic and conventional ASA-/mice was reduced by 17 and 22 percent, respectively (Figure 2D; $\mathrm{F}_{2,28}=$ 6.277, $P<0.01$ ANOVA). No significant difference between the two groups of ASA-/- mice was detectable.

\section{General Phenotype of Transgenic ASA-/- Mice}

To detect possible effects of the transgene expression on the general phenotype, the body weight, organ weight, lethality, litter size, litter frequency, and gender ratio was determined. No significant differences were detectable be- 

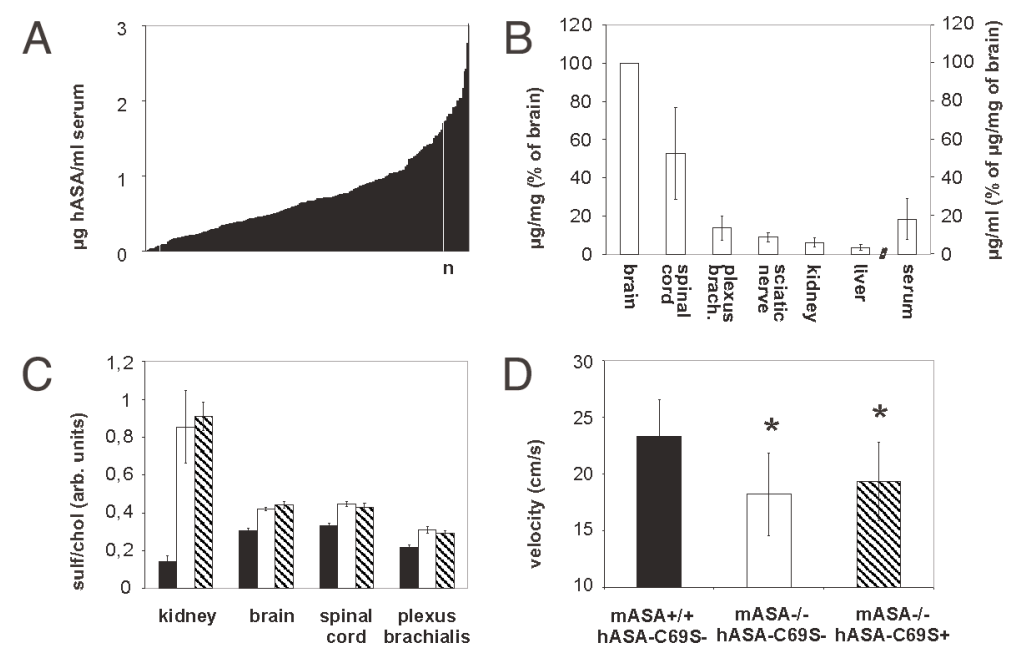

Figure 2. Transgene expression and MLD-like phenotype of transgenic ASA-/- mice. Bars indicate means $\pm S D$. (A) hASA-C69S concentrations in serum of 234 transgenic ASA-/mice. hASA-C69S was undetectable in eight mice due to serum levels below $0.01 \mu \mathrm{g} / \mathrm{mL}$. (B) Tissue distribution of hASA-C69S in mice with serum levels between 0.2 and $0.9 \mu \mathrm{g} / \mathrm{mL}$. Tissue and serum levels were normalized on hASA-C69S levels of brain. $n=5$. (C) Sulfatide concentration in tissues of six-month-old wildtype control mice (closed bars), non-transgenic ASA-/- mice (open bars) and transgenic ASA-/- mice (hatched bars; hASA-C69S serum level: 0.2-0.9 $\mu \mathrm{g} / \mathrm{mL}$ ). Sulfatide was quantified by TLC and normalized on the cholesterol concentration. $n=5$. (D) Swimming velocity. $n=10 .{ }^{*} P<0.05$ compared with wildtype controls. hASA-C69S serum levels of transgenic ASA-/- mice: 0.2-3.1 $\mu \mathrm{g} / \mathrm{mL}$.

tween transgenic ASA-/- mice, conventional ASA-/- mice, and wildtype controls (Figure 4A-D).

Overexpression of a lysosomal sulfatase might induce depletion of the formylglycine-generating enzyme (FGE), an activator of various sulfatases (21). This may result in reduced activities of sulfatases $(11,23)$. To analyze a possible inhibitory effect of hASAC69S expression, the compound sulfatase activity of serum was measured (Figure 2E). No significant difference could be detected between transgenic and non-transgenic mice.

Overexpression of a lysosomal enzyme may further cause depletion of mannose 6-phosphate (M6P) receptors in the trans Golgi network resulting in the hypersecretion of newly synthesized soluble lysosomal enzymes (23). To detect a possible misrouting of acidic hydrolases, the serum activity of four lysosomal enzymes was analyzed (Figure 4F). None of the four enzymes showed any groupspecific alterations.

\section{Lysosomal Morphology in Mice Expressing the Transgene on the Wildtype Background}

To investigate whether hASA-C69S expression induces a morphologically detectable lysosomal phenotype, tissues were analyzed by electron microscopy. Possible lysosomal changes might be masked by sulfatide storage in ASA-/mice. Therefore, mice which expressed the transgene on the wildtype background were used for this investigation. Electron microscopy revealed a normal size of lysosomes in liver and kidney (Figure 3E and not shown). However, intralysosomal deposition of small amounts of glycogen particles was seen in several hepatocytes. Although glycogen storage was low and found only in a minority of lysosomes, it was significant and absent from wildtype controls.

\section{Immune Response to Enzyme Replacement with hASA}

To analyze immune responses to wildtype hASA, mice were challenged by weekly intravenous injection of recombinant hASA. Conventional ASA-/- mice treated with $20 \mathrm{mg} / \mathrm{kg}$ hASA developed hypersensitivity reactions after the third and fourth injection. The reactions were characterized by reduced cage activity and breathing difficulties, possibly leading to lethargy and death (Figure 5A). Treatment of this group was terminated after four injections due to a high mortality of 57 percent. Wildtype mice treated with $20 \mathrm{mg} / \mathrm{kg}$ hASA showed the same type of reactions. They emerged, however, later (for the first time after the fourth injection) and were substantially milder. Consequently, none of the treated animals died within ten weeks. In contrast to the two control groups, transgenic knockout mice treated with comparable $(15 \mathrm{mg} / \mathrm{kg})$ or even higher rhASA doses (50 mg/ kg), tolerated treatment for ten weeks without casualties and hypersensitivity reactions (Figure 5A and not shown).

In a second experiment, mice were treated for up to 17 weeks with $2.5 \mathrm{mg} / \mathrm{kg}$ hASA and $\alpha$-hASA antibodies were titered in weekly or biweekly intervals. The group of transgenic ASA-/- mice comprised animals without detectable hASA-C69S in serum $(<0.01 \mu \mathrm{g} / \mathrm{mL}$, $\mathrm{n}=2$ ) and mice with low to high serum levels $(0.07-1.70 \mu \mathrm{g} / \mathrm{mL}, \mathrm{n}=10)$. Conventional ASA-/- mice showed progressive hypersensitivity/anaphylactic reactions and 25 percent of the animals died within four weeks (not shown). Concomitantly, the mice developed high titers of $\alpha$-hASA antibodies (Figure 5B). Increasing levels of $\alpha$-hASA antibodies were also detectable in wildtype controls, however, no animal was lost within the first ten weeks of treatment. Two mice died, however, after injection 11 and 17. On the contrary, no increase of $\alpha$-hASA antibodies, no hypersensitivity/anaphylaxis and no loss of animals was detectable in any of the 12 transgenic ASA-/- mice.

\section{Therapeutic Effects of Long-Term, Low-Dose Enzyme Replacement in Transgenic ASA-/- Mice}

Intravenous injection of recombinant hASA reduced sulfatide storage in con- 

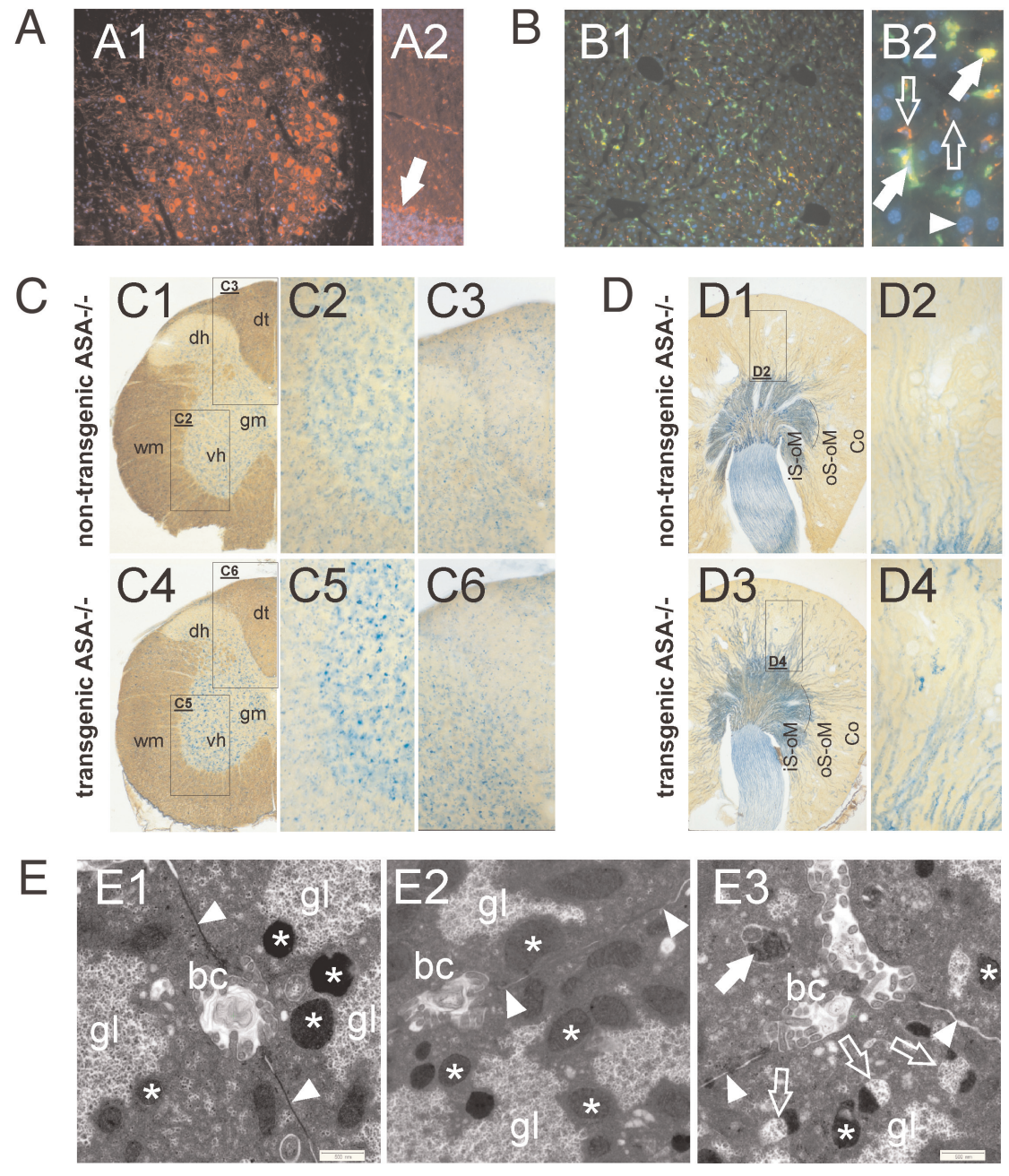

wildtype
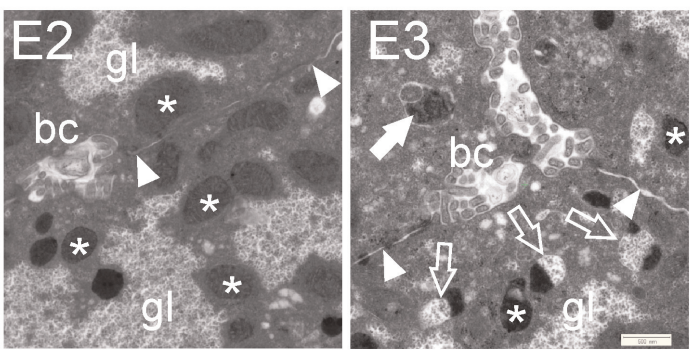

transgenic ASA+/+

Figure 3. Histology at age 11-12 months (A) hASA-C69S expression in the CNS of transgenic ASA-/- mice. Immunostaining of brain stem (A1) and cerebellum (A2) detects high hASA-C69S levels in neuronal perikarya of brain stem nuclei and the Purkinje cell layer (arrow). (B) Co-immunostaining of hASA-C69S (red) and F4/80 (green) reveals low transgene expression levels in liver (B1). Higher magnification (B2) detects expression in some F4/80-negative (open arrows) and -positive cells (closed arrows) with small nuclei, but not in hepatocytes (one nucleus is indicated by an arrowhead). (C) Cervical spinal cord of a conventional (C1-C3) and a transgenic ASA-/- mouse (C4-C6). Sections (100 $\mu \mathrm{m}$ ) were incubated with alcian blue to visualize sulfatide storage. C1, C4-overviews to outline the regions shown at higher magnification. gm - gray matter, wm - white matter, dh - dorsal horn, dt - dorsal tract, vh - ventral horn. (D) Alcian blue-incubated sections (100 $\mu \mathrm{m}$ ) through kidney of a conventional (D1, D2) and a transgenic ASA-/- mouse (D3, D4). D1, D3 - overview; regions shown at higher magnification are boxed. Storage material is seen in the thick ascending limbs of Henle's loop (TAL) of the inner (iS-OM) and outer stripe of the outer medulla (OS-OM), and to a lesser extent in the TALs of the medullary rays of the cortex (Co). D2, D4 - cortex. (E) Electron micrographs of hepatocytes. E1 - wildtype control mouse; E2, E3 - transgenic mouse expressing hASA-C69S on the wildtype background (serum level $0.91 \mu \mathrm{g} / \mathrm{mL}$ ). Most lysosomes of the transgenic mouse show a normal morphology (asterices). However, some contain glycogen particles (open arrows in E3). Autophagic vacuoles do not contain glycogen (closed arrow). gl - cytosolic glycogen, bc bile canaliculus, arrowheads - cell boundary.

ventional ASA-/- mice (6). We speculated that a high extracellular level of hASA-C69S may interfere with M6Pdependent endocytosis and lysosomal targeting of therapeutic enzyme due to competition with $\mathrm{M} 6 \mathrm{P}$ receptors and/or other components of the endocytic pathway. To detect a possible inhibition of metabolic correction, transgenic ASA-/mice were treated with hASA and sulfatide levels were determined. To favor competitive effects we used mice showing high hASA-C69S levels and low enzyme doses. In detail, $2.5 \mathrm{mg} / \mathrm{kg}$ hASA was injected in weekly intervals for 17 weeks into three transgenic mice with hASA-C69S serum levels between 1.27 and $1.70 \mu \mathrm{g} / \mathrm{mL}$. As a control, three mice with undetectable to intermediate serum levels of $<0.01,0.38$, and $0.66 \mu \mathrm{g} / \mathrm{mL}$ were treated. Considered as a group, the mean sulfatide levels of the six mice declined significantly in kidney, spinal cord, and plexus brachialis, but not in the brain (Figure 6). Looking at individual mice, no correlation between the extent of sulfatide reduction and the hASA-C69S serum concentration was obvious. In the kidney, for example, residual sulfatide levels were lowest and highest in two mice with serum levels of 1.27 and $<0.01 \mu \mathrm{g} / \mathrm{mL}$, respectively (not shown).

\section{Discussion}

Previous studies indicated that repeated treatment of ASA-/- mice with recombinant hASA substantially reduces intralysosomal sulfatide storage (6). Surprisingly, treatment also was effective in improving the pathology and function of the CNS. It was concluded that ERT may be a promising therapeutic option in this devastating lysosomal storage disease for which curative treatment is not available to date. The preclinical studies in mice were, however, compromised by a progressive humoral immune response to repeatedly injected hASA. Two types of immune-mediated side effects were discernible (Matzner et al., submitted). First, the development of treatment resistance which becomes apparent by the reaccu- 

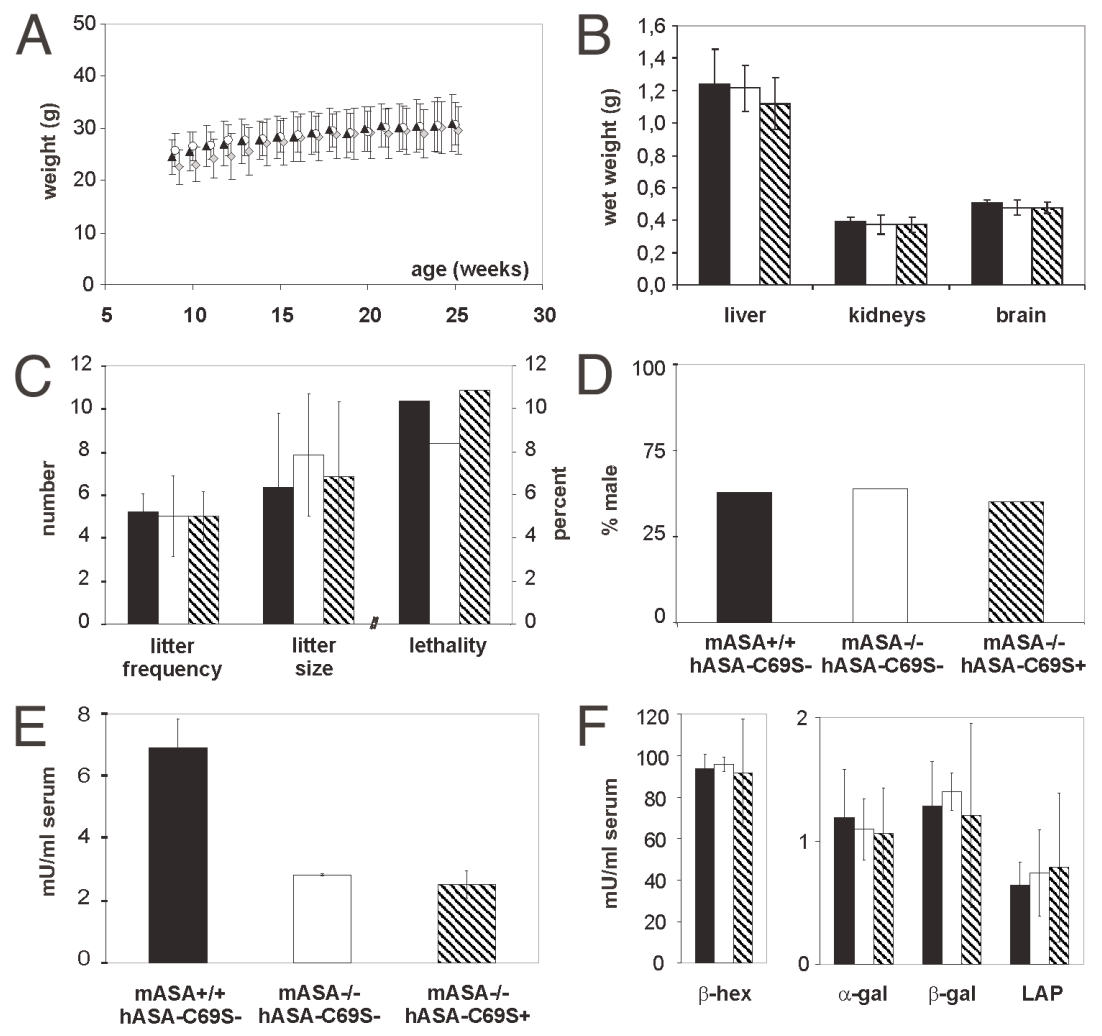

Figure 4. General phenotype of transgenic ASA-/- mice (hatched bars and symbols), nontransgenic ASA-/- mice (open bars and symbols) and wildtype mice (closed bars and symbols). Bars and points indicate means \pm SDs. (A) Body weight. $n=10$. (B) Organ weights at age 6 mo. $n=10$. (C) Littering. The litter frequency indicates the total number of litters produced by five to seven permanent breeding pairs mated at age five to nine weeks for six months. Litter sizes and lethality before age five weeks was evaluated for 33-64 litters. (D) Fraction of male pups among 83-363 newborns. (E) Activity of sulfatases in serum. $n=3$. hASA-C69S serum levels of transgenic ASA-/- mice: 0.3-0.7 $\mu \mathrm{g} / \mathrm{mL}$. (F) Serum activities of $\beta$-hexosaminidase ( $\beta$-hex), $\alpha$-galactosidase ( $\alpha$-gal), $\beta$-galactosidase ( $\beta$-gal) and lysosomal acid phosphatase (LAP). Same mice as in (E).

mulation of transiently diminished sulfatide levels after prolonged treatment. At least three antibody-mediated processes contributed to treatment resistance: redistribution of substituted hASA from sulfatide-storing cells to macrophages, inhibition of mannose 6phosphate-dependent internalization of enzyme, and intracellular misrouting of endocytosed hASA. Activity-inhibiting antibodies were not detectable. The second type of immune-mediated side effects were anaphylactic reactions causing the death of animals. As a consequence of the immune complications, the full therapeu- tic potential of ERT has not been evaluated so far.

To create an MLD mouse model which tolerates long-term treatment, we adapted a strategy initially used by Sly and coworkers to induce immune tolerance in the MPS VII mouse model (9). An inactive mutant of hASA was constitutively expressed from a transgene stably integrated into the ASA-/- background. Inactivation of hASA was achieved by exchanging an essential active site cysteine by serine (Figure 1) (20). Although this mutation abrogates the catalytic activity of hASA, it seems not to alter the three dimensional folding and epitope pattern of the polypeptide. Thus, antibodies raised against wildtype hASA still recognize the mutant hASA polypeptide in ELISA and immune fluorescence experiments (Figure $2 \mathrm{~A}, \mathrm{~B}, 3 \mathrm{~A}$ ). Normal folding also is suggested by the absence of hASA-C69S accumulation in the endoplasmic reticulum (Figure 1D), which is observed for many other hASA mutants due to the retention by the conformational proof-reading system of the cell (16).

A surprisingly high variation of hASAC69S expression was detectable between individual transgenic mice (Figure 2A). This may reflect variations in the effectiveness of promoter inactivation by methylation or differences in transgene copy numbers. Possibly, multiple integration sites in the original founder segregated during breeding of F2 and F3 mice used in this study. Integration into different chromosomes is, however, unlikely because in all generations transgenic mice were born in frequencies according to Mendelian rules for monogenic inheritance. Alternatively, a single integration of an initial high-copy tandem repeat was expanding or shrinking in subsequent generations by unequal crossover or deletion. A detailed analysis of transgene copy numbers and integration site(s) would be required to address these notions. Compared with the endogenous murine ASA levels in wildtype mice (14), hASA-C69S expression ranged between $<0.06$ - to 31-fold, $<0.08$ to 23 fold and $<0.73$ to 225 -fold of normal for serum, liver, and brain, respectively (Figure 2 A,B). We exploited the availability of transgenic mice with widely differing transgene expression levels by analyzing possible dose-dependent effects of transgene expression.

We first speculated that high levels of hASA-C69S may induce a new phenotype because an overexpressed lysosomal enzyme may deplete factors which are required for transport and/or modification of other lysosomal enzymes (23). The occurrence of gross phenotypical changes could, however, be excluded by an extensive analysis of the biochemistry, histology, behavior and reproduction of 

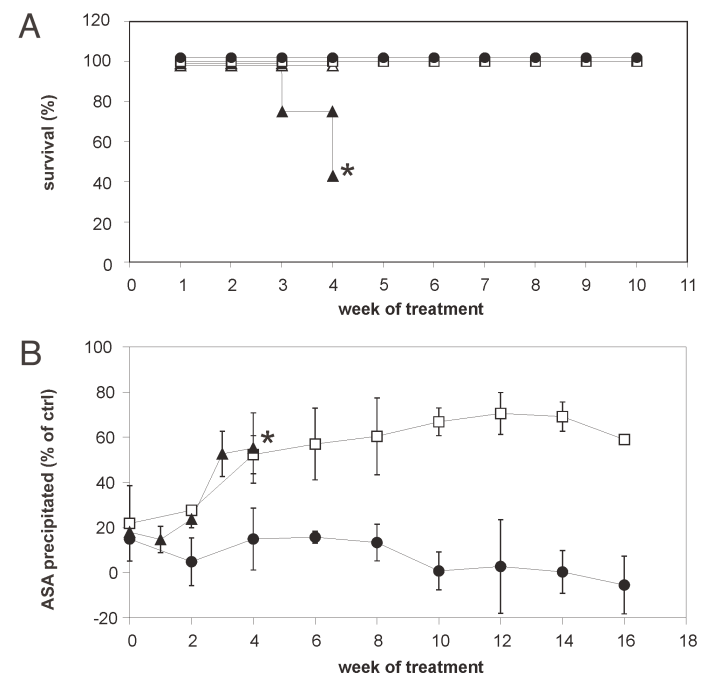

Figure 5. Immune responses of mice treated by weekly injection of hASA. (A) Lethality during treatment for up to ten weeks using $20 \mathrm{mg} / \mathrm{kg}$ (conventional ASA-/- mice (closed triangles, $n=20$ ) and wildtype controls (open squares, $n=5$ )) or $15 \mathrm{mg} / \mathrm{kg}$ hASA (transgenic ASA-/- mice (closed circles, $n=20$ )). As a control, some conventional ASA-/- mice were treated with buffer (open triangles, $n=10$ ). Asterisk- due to severe side effects treatment was terminated after four injections. (B) $\alpha$-hASA antibody titers of mice treated for up to 17 weeks with $2.5 \mathrm{mg} / \mathrm{kg}$ hASA. Same legend as in (A). hASA-C69S serum concentrations of transgenic ASA-/- mice: < 0.01-1.70 $\mu \mathrm{g} / \mathrm{mL}$. Bars indicate means $\pm \mathrm{SDs}, \mathrm{n}=4-5$. transgenic ASA-/- mice (Figures 2-4). Notably, overexpression of hASA-C69S did not diminish the compound sulfatase activity indicating that FGE is not depleted by the transgene product

(Figure 4E). This is in contrast to a previous study, in which constitutive expression of an active site mutant of another lysosomal sulfatase (N-acetylgalactosamine6-sulfate sulfatase) reduced the activities of endogenous sulfatases by five to 30 percent (11). Differences in the transgene expression levels and/or affinities of the transgene products to FGE may account for this discrepancy. Electron microscopy unexpectedly revealed intralysosomal

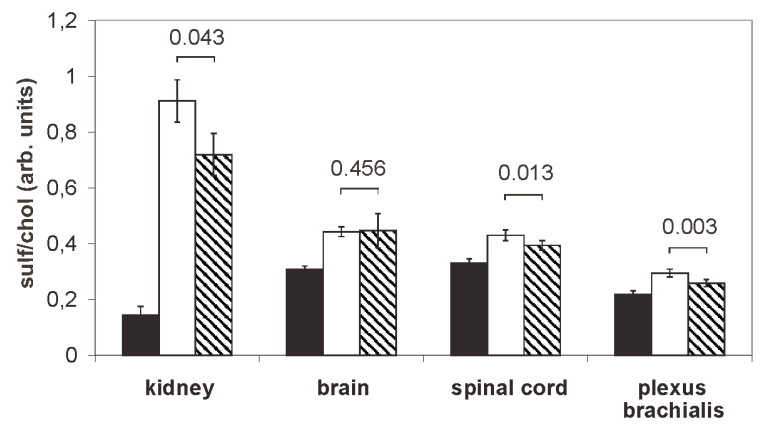

Figure 6. Sulfatide levels after weekly injection of $2.5 \mathrm{mg} / \mathrm{kg}$ hASA into transgenic ASA-/mice for 17 weeks (hatched bars; hASA serum levels $<0.01-1.70 \mu \mathrm{g} / \mathrm{mL}$ ). Age-matched transgenic ASA-/- mice mock-treated with buffer (open bars) and untreated wildtype mice (closed bars) were used as controls. Sulfatide was quantified by TLC and normalized on cholesterol. Bars represent means $\pm S D s, n=5-6$. $P$ values are indicated (Student $t$-test). glycogen storage in some hepatocytic lysosomes (Figure 3E). Storage did by no means reach the extent of glycogen storage in Pompe disease, a lysosomal storage disorder caused by deficiency of $\alpha$ glucosidase (24). Furthermore, no storage was detectable in other tissues like collecting ducts of kidney where glycogen storage is prominent in inherited or induced $\alpha$-glucosidase deficiency (not shown). The deviation from the storage pattern typical for Pompe disease argues against $\alpha$-glucosidase inactivation for example, by insertion mutagenesis. It rather suggests subtle and cell typespecific effects of hASA-C69S expression on the lysosomal homeostasis. The question of how hASA-C69S can induce a new lysosomal phenotype is particularly important to predict side effects of gene therapy approaches which aim at a high level overexpression of hASA from strong viral promotors.

In a second set of experiments, we analyzed whether transgene expression confers tolerance to wildtype hASA and, if so, which expression level is required for tolerance induction. For this purpose, mice with widely differing transgene expression levels were treated by weekly injection of high (15-20 mg/kg) or low hASA doses $(2.5 \mathrm{mg} / \mathrm{kg})$. Similar to previous preclinical studies (6) non-transgenic ASA-/- mice rapidly developed lethal anaphylactic reactions and high titers of $\alpha$-hASA antibodies (Figure 5). Transgenic ASA-/- mice, on the contrary, were entirely protected from immune complications and treatment neither induced antibody expression nor hypersensitivity reactions. Importantly, even transgenic mice with serum levels below the limit of detection were unresponsive, demonstrating that immunological tolerance was independent of the level of transgene expression. Our observations confirm data from other LSD mouse models in which sub- or supranormal levels of inactive enzyme (9-11) as well as minute amounts of active enzyme (25) effectively precluded deleterious immune responses. Furthermore, wildtype mice also were partially protected from immune complications to 
hASA (Figure 5A). Consequently, even a polypeptide which is structurally related to hASA, namely the murine ASA sharing 86 percent identity on the amino acid level (26), can protect to some extent from anaphylactic reactions. The reduced responsiveness of wildtype mice is likely to reflect the smaller number of epitopes to which the mouse can react, because they are not shared by murine and human ASA. The fact that both a structurally related ASA variant as well as a very low residual hASA expression has protective effects in mice, suggests that MLD patients with cross-reacting immunological material may be protected from severe immune complications to ERT as well. Around 94 percent of MLD patients belong to this group (4).

Long-term treatment of transgenic ASA-/- mice with low hASA doses (17 $x$ $2.5 \mathrm{mg} / \mathrm{kg}$ ) reduced excess sulfatide in kidney, plexus brachialis, and spinal cord (Figure 6). Thus, similar to short-term, high-dose treatment $(4 \times 20 \mathrm{mg} / \mathrm{kg})$, storage was effective in reducing storage of the PNS and CNS (6). This argues against the notion that high extracellular levels of hASA-C69S interfere with metabolic correction, for example, by saturation of endocytosing cell surface receptors. In contrast to the previous study (in which the spinal cord was not analyzed), sulfatide was, however, not reduced in brain. Why the spinal cord, but not the brain responded to low-dose, long-term treatment is unclear and needs further investigation. It is, however, striking, that in all LSD mouse models in which reduction of brain storage has been achieved so far, high enzyme doses were required (27-30). This has been attributed to the paucity of mannose 6phosphate receptors on apical membranes of endothelial cells mediating transcytosis of therapeutic enzyme across the blood-brain barrier (31). Data from non-transgenic ASA-/- mice indicate that spinal cord acquires four-fold higher hASA levels than brain upon ERT (Matzner, unpublished). Therefore, the regional differences in the therapeutic efficacy might be ascribed to discrepancies in the tissue-specific uptake rates of hASA. Clearly, it would be of interest to determine therapeutic effects of sustained substitution of high hASA doses. In a still ongoing study, immune tolerant mice are therefore treated with 52 doses of 15 and $50 \mathrm{mg} / \mathrm{kg}$ hASA. The evaluation of biochemical, histological, electrophysiological, and behavioral effects will allow for the assessment of the full therapeutic potency of ERT for MLD.

In summary, we have generated an optimized mouse model of MLD by transgenic expression of an inactive hASA mutant on the ASA-/- background. Transgene expression does not alter the MLD-like phenotype, but confers absolute immune tolerance to wildtype hASA. The novel mouse strain represents MLD variants with residual enzyme levels, which account for the vast majority of MLD cases. In contrast to conventional ASA-/- mice, the new strain allows for long-term ERT in the absence of immunological side effects.

\section{ACKNOWLEDGMENTS}

The authors thank Dagmar Niemeier for excellent technical assistance and William Sly (St. Louis, MO, USA) for providing the plasmid pTVC. This work was supported by the European Leukodystrophy Association, ELA.

\section{REFERENCES}

1. von Figura K, Gieselmann V, Jaeken J. (2001) Metachromatic leukodystrophy. In Scriver CR, Beaudet AL, Sly WS, Valle D, Childs B, Kinzler KW, Vogelstein B. (eds.). The Metabolic and Molecular Bases of Inherited Disease. Mc Graw-Hill. New York, USA. pp. 3695-724.

2. Hess B et al. (1996) Phenotype of arylsulfatase A-deficient mice: relationship to human metachromatic leukodystrophy. Proc. Natl. Acad. Sci. U S A. 93:14821-6.

3. Sevin C, Aubourg P, Cartier N. (2007) Enzyme, cell and gene-based therapies for metachromatic leukodystrophy. J. Inherit. Metab. Dis. 30:175-83.

4. Polten A, Fluharty AL, Fluharty CB, Kappler J von Figura K, Gieselmann V. (1991) Molecular basis of different forms of metachromatic leukodystrophy. N. Engl. J. Med. 324:18-22.

5. Kyewski B, Klein L. (2006) A central role for central tolerance. Annu. Rev. Immunol. 24:571-606.

6. Matzner U et al. (2005) Enzyme replacement improves nervous system pathology and function in a mouse model for metachromatic leukodys- trophy. Hum. Mol. Genet. 14:1139-52.

7. Brooks DA. (1999) Immune response to enzyme replacement therapy in lysosomal storage disorder patients and animal models. Mol. Genet. Metab. 68: 268-75.

8. Brooks DA, Kakavanos R, Hopwood JJ. (2003) Significance of immune response to enzymereplacement therapy for patients with a lysosomal storage disorder. Trends Mol. Med. 9:450-3.

9. Sly WS et al. (2001) Active site mutant transgene confers tolerance to human beta-glucuronidase without affecting the phenotype of MPS VII mice. Proc. Natl. Acad. Sci. U. S. A. 98:2205-10.

10. Tomatsu $S$ et al. (2003) Production of MPS VII mouse (Gus(tm(hE540A x mE536A)Sly)) doubly tolerant to human and mouse beta-glucuronidase. Hum. Mol. Genet. 12: 961-73.

11. Tomatsu $S$ et al. (2005) Development of MPS IVA mouse (Galnstm(hC79S.mC76S)slu) tolerant to human N-acetylgalactosamine-6-sulfate sulfatase. Hum. Mol. Genet. 14: 3321-35.

12. Matzner U, Habetha M, Gieselmann V. (2000) Retrovirally expressed human arylsulfatase A corrects the metabolic defect of arylsulfatase A-deficient mouse cells. Gene Ther. 7:805-12.

13. Baum H, Dodgson KS, Spencer B. (1959) The assay of arylsulphatases A and B in human urine. Clin. Chim. Acta. 4: 453-5.

14. Matzner U, Harzer K, Learish RD, Barranger JA, Gieselmann V. (2000) Long-term expression and transfer of arylsulfatase A into brain of arylsulfatase A-deficient mice transplanted with bone marrow expressing the arylsulfatase A cDNA from a retroviral vector. Gene Ther. 7:1250-7.

15. Stein C et al. (1989) Cloning and expression of human arylsulfatase A. J. Biol. Chem. 264:1252-9.

16. Poeppel P, Habetha M, Marcao A, Bussow H, Berna L, Gieselmann V. (2005) Missense mutations as a cause of metachromatic leukodystrophy. Degradation of arylsulfatase A in the endoplasmic reticulum. FEBS J. 272:1179-88.

17. Klein D, Bussow H, Fewou SN, Gieselmann V. (2005) Exocytosis of storage material in a lysosomal disorder. Biochem. Biophys. Res. Commun. 327:663-7.

18. D'Hooge R, Van Dam D, Franck F, Gieselmann V, De Deyn PP. (2001) Hyperactivity, neuromotor defects, and impaired learning and memory in a mouse model for metachromatic leukodystrophy. Brain Res. 907:35-43.

19. Matzner U et al. (2002) Bone marrow stem cellbased gene transfer in a mouse model for metachromatic leukodystrophy: effects on visceral and nervous system disease manifestations. Gene Ther. 9:53-63.

20. Dierks T, Schmidt B, von Figura K. (1997) Conversion of cysteine to formylglycine: A protein modification in the endoplasmic reticulum. Proc. Natl. Acad. Sci. U.S.A. 94:11963-8.

21. Wittke D, Hartmann D, Gieselmann V, LullmannRauch R. (2004) Lysosomal sulfatide storage in the brain of arylsulfatase A-deficient mice: cellular alterations and topographic distribution. Acta 
Neuropathol. (Berl). 108:261-71.

22. Lullmann-Rauch R, Matzner U, Franken S,

Hartmann D, Gieselmann V. (2001) Lysosomal sulfoglycolipid storage in the kidneys of mice deficient for arylsulfatase A (ASA) and of double-knockout mice deficient for ASA and galactosylceramide synthase. Histochem. Cell Biol. 116: 161-9.

23. Matzner U, Gieselmann V. (2005) Gene therapy of metachromatic leukodystrophy. Expert Opin. Biol. Ther. 5:55-65.

24. Hirschhorn R, Reusser AJ. (2000) Glycogen storage disease type II. In Scriver CR, Beaudet AL, Sly WS, Valle D, Childs B, Kinzler KW, Vogelstein B (eds.). The Metabolic and Molecular Bases of Inherited Disease. Mc Graw-Hill. New York, USA. pp. 3389-420.

25. Raben $\mathrm{N}$ et al. (2003) Induction of tolerance to a recombinant human enzyme, acid alpha-glucosidase, in enzyme deficient knockout mice. Transgenic Res. 12:171-8.

26. Kreysing J et al. (1994) Structure of the mouse arylsulfatase A gene and cDNA. Genomics. 19: 249-56.

27. Lee WC et al. (2005) Enzyme replacement therapy results in substantial improvements in early clinical phenotype in a mouse model of globoid cell leukodystrophy. FASEB J. 19:1549-51.

28. Roces DP et al. (2004) Efficacy of enzyme replacement therapy in alpha-mannosidosis mice: a preclinical animal study. Hum. Mol. Genet. 13: 1979-88.

29. Dunder U et al. (2000) Enzyme replacement therapy in a mouse model of aspartylglycosaminuria. FASEB J. 14:361-7.

30. Vogler C et al. (2005) Overcoming the bloodbrain barrier with high-dose enzyme replacement therapy in murine mucopolysaccharidosis VII. Proc. Natl. Acad. Sci. U. S. A. 102:14777-82.

31. Urayama A, Grubb JH, Sly WS, Banks WA. (2004) Developmentally regulated mannose 6-phosphate receptor-mediated transport of a lysosomal enzyme across the blood-brain barrier. Proc. Natl. Acad. Sci. U. S. A. 101:12658-63. 